\title{
Morphology and surface topography of Ti6Al4V lattice structure fabricated by selective laser sintering
}

\author{
J. MASZYBROCKA ${ }^{1 *}$, A. STWORA ${ }^{2}$, B. GAPIŃSKI ${ }^{3}$, G. SKRABALAK ${ }^{2}$, and M. KAROLUS ${ }^{1}$ \\ ${ }^{1}$ University of Silesia, Institute of Materials Science, ul. 75 Pułku Piechoty 1A, 41-500 Chorzów \\ ${ }^{2}$ Institute of Advanced Manufacturing Technology, ul. Wrocławska 37A, 30-011 Kraków \\ ${ }^{3}$ Poznań University of Technology, Institute of Mechanical Technology ul. Piotrowo 3, 60-965 Poznań
}

\begin{abstract}
Construction of metallic implants with a porous structure that mimics the biomechanical properties of bone is one of the challenges of orthopedic regenerative medicine. The selective laser sintering technique (SLS) allows the production of complex geometries based on three-dimensional model, which offers the prospect of preparing porous metal implants, in which stiffness and porosity can be precisely adjusted to the individual needs of the patient. This requires an interdisciplinary approach to design, manufacturing and testing of porous structures manufactured by selective sintering. An important link in this process is the ability to assess the surface topography of the struts of porous structure. The paper presents a qualitative assessment of the surface morphology based on SEM studies and methodology that allows for quantitative assessment of stereometric structure based on micro-tomographic measurements.
\end{abstract}

Key words: metal porous implant, selective laser sintering, surface roughness, X-ray microtomography.

\section{Introduction}

Titanium and its alloys are widely used for the manufacture of medical implants. They owe their popularity to a combination of favorable properties, i.e. good mechanical properties, relatively low density and outstanding biocompatibility as compared to other metallic materials [1]. Unfortunately, their lifetime in the body is limited. In case of metallic implants with solid structure it is related to their higher stiffness in comparison with bone tissue. Disorders of the distribution of stresses and strains in the structure of the bone tissue surrounding the implant lead to bone resorption around the implant and may result in aseptic loosening of the implant $[2,3]$. The use of porous implant may help to solve this problem [4]. Good and durable connection of the implant to the bone tissue is possible when there are provided sufficient conditions for the ingrowth of bone into the pores of the material. An important role in this process plays the geometric properties of porous implant, i.e. size and shape of the pores, ways of connection between them. Studies indicate that the optimum pore size should be in the range of 100-700 $\mu \mathrm{m}$. The pores should be open and the degree of porosity should be $60-80 \%[5,6]$. The search for an optimal model of spatial architecture in a porous implant must also take into consideration aspects of the strength of the implant, which practically determines the range of porosity and pore size in the structure.

There are many conventional methods for preparing porous metallic structures, i.e. powder metallurgy with space holder, compression and fiber sintering, sponge replication, freeze casting method [7-11]. None of these methods allows to ob-

\footnotetext{
*e-mail: joanna.maszybrocka@us.edu.pl
}

tain an implant with a defined pore structure. An alternative to traditional methods of manufacturing metallic porous material is rapid prototyping by selective laser sintering (SLS) or selective electron beam melting (EBM). The ability to produce complex geometric shapes based on three-dimensional CAD model allows for precise control of not only the product geometry itself, but also of shape, size and distribution of pores in the proposed structure.

Taking into account requirements set for porous medical implants, design, manufacturing and testing of porous structures manufactured by SLS require an interdisciplinary approach. An important role in this process is played, among other things, by verification of the accuracy of the porous implant $3 \mathrm{~d}$ geometry and the evaluation of the surface topography of the struts. Nowadays a large number of instruments for measuring surface roughness is available [12]. Most often they operate on the basis of the profile method. Data is collected based on the points arranged in a line or based on image registration from different positions. The choice of specific method is determined by the purpose and conditions of measurement $[13,14]$. The measurement of surface roughness inside the porous structures manufactured by selective laser sintering is practically not achievable (without destroying the object) by conventional methods. An alternative is constituted by $x$-ray microtomography. The authors' $[15,16]$ work studies have shown that in case of a relatively large surface roughness (micro-scale) a comparable, or even better accuracy can be achieved using the mentioned method than with conventional measuring methods. For the measurements of small surface roughness (sub-micronscale) limitation may constitute spatial resolution of the images and noise. The aim of this study is to investigate the morphology and the surface topography of the Ti6A14V porous structure produced by selective laser sin- 
tering. Morphology makes qualitative evaluation of the three dimensional shape of a surface available, whereas topography provides quantitative feature dimensions.

\section{Experimental details}

2.1. Lattice structure modelling. A surface belonging to the family of triply periodic minimal surface (TPMS) was selected for modeling. TPMS are smooth infinite surfaces that partition space into two intertwined labyrinth that are periodic in three independent directions [17]. The chosen model is described by the equation:

$$
\begin{aligned}
& k_{1} \cdot(\sin (x) \cdot \sin (y) \cdot \sin (z)+\sin (x) \cdot \cos (y) \cdot \cos (z) \\
& +\cos (x) \cdot \sin (y) \cdot \cos (z))+\cos (x) \cdot \cos (y) \cdot \sin (z) \\
& +k_{2} \cdot(\cos (4 \cdot x)+\cos (4 \cdot y)+\cos (4 \cdot z))+k_{3}=0
\end{aligned}
$$

The model parameters $\left(\mathrm{k}_{1}, \mathrm{k}_{2}, \mathrm{k}_{3}\right)$ were chosen to achieve the porosity of the structure $\mathrm{Vv}=80 \%$. The volume ratio defined as the ratio of the volume of material to the total volume of the surrounding area. It was assumed that the area of modeling will constitute a cube with dimensions of $15 \times 15 \times 15 \mathrm{~mm}$. The three-dimensional mesh as a set of coordinates of the vertices and the set of elements that define the topology of the grid has been exported to *.obj format. The elementary cell and the finished model is shown in Fig. 1. The design process

a)

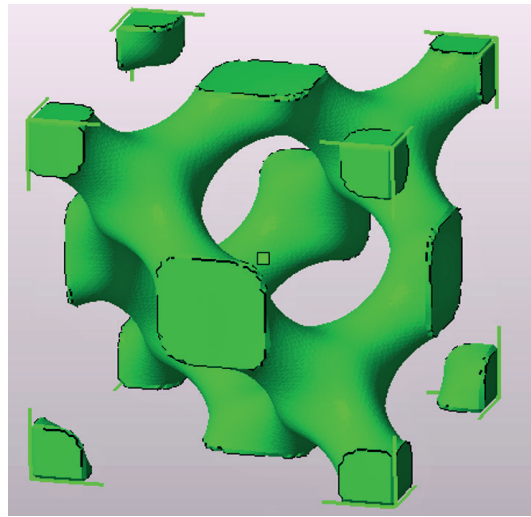

b)

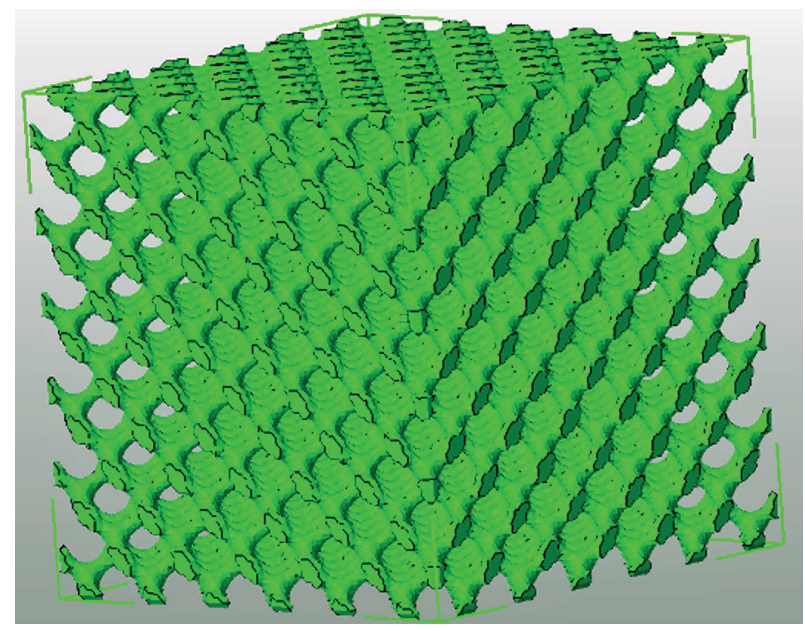

Fig. 1. The basic unit cell (a) and the generated model of the lattice (b) of the $3 \mathrm{~d}$ model was performed in the Matlab software and the final preparation of the model to the manufacturing process by selective laser sintering was performed in the Marcam Autofab software.

2.2. Research material. The porous structure was manufactured from Ti6Al4V powders (Renishaw). The powders have the spherical shape, their surface was smooth. This indicates a good flowability. The average diameter of the powder was $40 \mu \mathrm{m}$ (Fig. 2). The chemical composition of the powders specified by the manufacturer are summarized in Table 1.

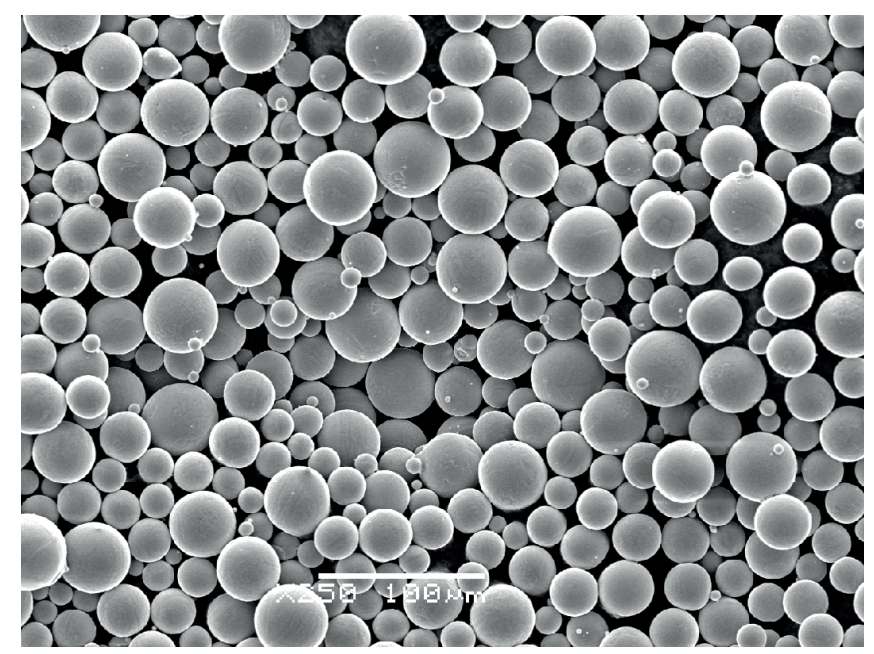

Fig. 2. Ti6Al4V powder (SEM)

Table 1

Composition of powder Ti6Al4v

\begin{tabular}{|l|c|c|c|c|c|}
\hline Element & $\mathrm{Ti}$ & $\mathrm{Al}$ & $\mathrm{V}$ & $\mathrm{Fe}$ & $\mathrm{O}$ \\
\hline Mass [\%] & Balance & $5.50-6.50$ & $3.50-4.50$ & $<0.25$ & $<0.13$ \\
\hline Element & $\mathrm{C}$ & $\mathrm{N}$ & $\mathrm{H}$ & \multicolumn{2}{|c|}{ Residual } \\
\hline Mass [\%] & $<0.08$ & $<0.05$ & $<0.012$ & \multicolumn{2}{c|}{$<0.10$ each } \\
\hline
\end{tabular}

2.3. Selective laser sintering. The $3 \mathrm{~d}$ model was manufactured by selective laser sintering techniques on the AM $250 \mathrm{Re}-$ nishaw device, equipped with a $400 \mathrm{~W}$ fiber laser $\mathrm{Yb}$-fiber $(\lambda=1064 \mathrm{~nm})$. The parameters of the sintering process are summarized in Table 2 . The chamber keeps oxygen concentrations below 50 ppm.

Table 2

The parameters of the sintering process

\begin{tabular}{|c|c|c|}
\hline Laser power & Scan speed & Hatch spacing \\
\hline $400 \mathrm{~W}$ & $150 \mathrm{~mm} / \mathrm{s}$ & $75 \mathrm{~mm}$ \\
\hline Layer thickness & Scan strategy & \\
\hline $50 \mathrm{um}$ & meander & \\
\hline
\end{tabular}




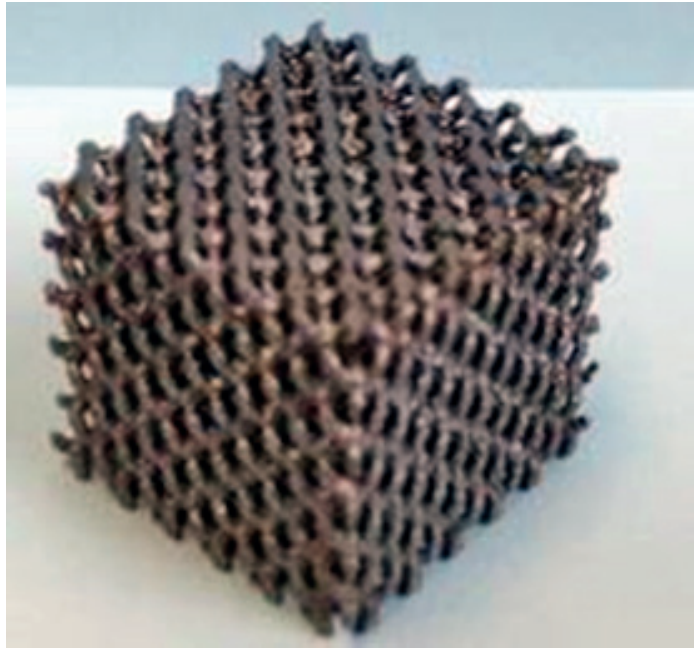

Fig. 3. Porous Ti6Al4V fabricated by selective laser sintering (SLS)

The porous structure with dimensions of $15 \times 15 \times 15 \mathrm{~mm}$ was cut off from the base plate, on which has been manufactured, using Electrical Discharge Machining (EDM). In order to remove residual powder from the sintering process, the samples were pre-treated: flow of compressed air then cleaned in an ultrasonic cleaner, in ethanol for 30 minutes.

2.4. SEM microscopy. Microscopic observations were performed on a scanning microscope JEOL JSM 6480's. To reflect the nature of three-dimensional structure of the sample the observations were performed at a tilted table (approximately 25 degrees).

2.5. X-ray microtomography. Tomographic studies were carried out on the measuring X-ray tomograph GE v|tome|x s 240. The device is equipped with two X-ray sources - the first one is a reflection target with a maximum power of $320 \mathrm{~W}$, the second one is a transmission target with maximum power of $15 \mathrm{~W}$. To the investigation both X-ray sources were used - the reflection target with micro focus allowed to examine the entire structure of Ti6Al4V made using 3D printing (Fig. 3). These studies were carried out in macro scale for the magnification $2.4 \mathrm{x}$ and a voxel size of $83 \mu \mathrm{m}$. The next step was to measure the structure of the tested piece using a nanofocus transmission target source. This solution allowed to receive in micro scale a tomographic image with $102 x$ magnification with a voxel size of $1.9 \mu \mathrm{m}$. This enables a much more accurate representation of the bar structure and in the morphology of the outer surface (Fig. 5).

\section{Analysis of the results}

3.1. Morphology of the struts surface on the basis of SEM observation. SEM observation of the struts surface shows their complex morphology (Fig. 4). The spherical Ti6Al4V powders
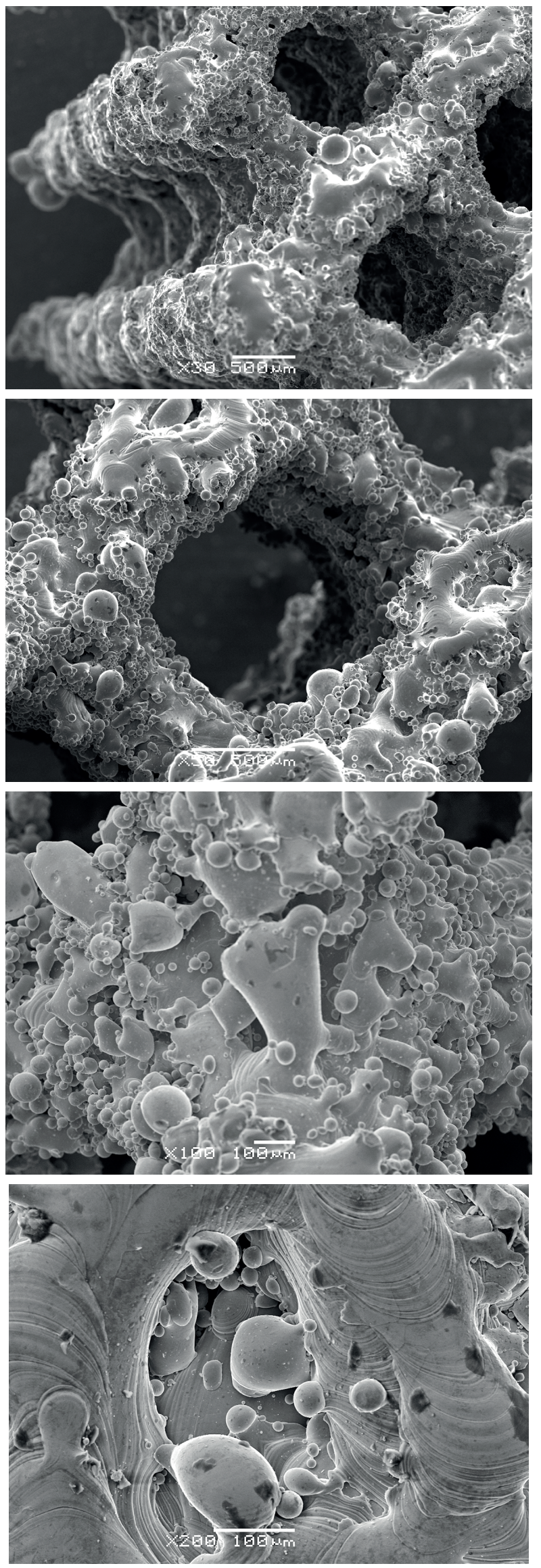

Fig. 4. The morphology of the struts in the Ti6Al4V porous lattice 
or partially melted agglomerates are attached to their surface. The presence of attached particles is a consequence of the method for producing the porous structures $[18,19]$. The large difference in temperature between the sintered material and the surrounding loose powder induces thermal diffusion phenomena which results in partial melting of the powder and its attachment to the struts. Part of the powders can be melted by the laser beam carried on the surface of the powder as previously marked path and then bonded to the boundary of each layer. In case of inclined struts the manufacturing process it is partly based on the previously sintered layer of material and the loose powder. Some of the metal particles below each layer may be totally or partially melted and then connected with a lower layer of material. This mechanism explains why the lower surfaces of the struts exhibit greater roughness than the upper part. On inclined struts there are visible characteristic steps as a result of the layer by layer sintering process. (Fig. 1c). The height of the steps is determined by the thickness of the applied layer of powder during the sintering process, which is one of the process parameters (Table 2).

3.2. Morphology of the struts surface on the basis of $x$-ray computer microtomography. The use of the CT scanner allowed for evaluation of the surface morphology of struts throughout the volume of the porous structure. Fig. 5 shows a three-dimensional visualization of the fragments of the struts. Just like in the outer layer, which was evaluated based on SEM observation, to the struts inside the porous structure also adhered partially melted spherical Ti6Al4V powder or agglomerates.

3.3. Quantitative analysis of the surface topography. An attempt at quantitative description of the struts surface topography was carried out on the basis of tomographic measurements. Measurement data was prepared in the VGstudio soft- ware, and the calculations were performed in MountainsMap software (DigitalSurf). In order to determine the 3D parameters of struts, it was necessary to process the data first. For this purpose a functionality of the MountainsMap software which allows read*.stl files (Binary Stereolithography Range Data) was used. Measured struts were rotated in such a way, that the area to be analyzed set in the planar view (viewed from a point on the positive $Z$ axis in the direction of the origin $(0,0,0)$ ). The result was the view of the XY plane (Fig. 6a). The measurement file prepared in such a way has been imported into MountainsMap software. As a result of interpolation performed by the software nonuniform cloud of points was converted onto a rectangular grid (Fig. 6b). For the analysis 8 areas located in different parts of the bar (Fig. 7) were chosen. For the analysis the areas of the struts which were not steeply inclined with respect to the XY plane were chosen (Fig. 6a). As a reference surface in relation to which the surface roughness parameters was calculated, a second degree polynomial were chosen. (Fig. 7). In the selection of reference surface the fact, that the selected areas are only a small part of the investigated surface was taken into account and the reference surface defined as second degree polynomial is the most common solution in this case [12].

Amplitude parameters calculations, taking into account 8 analyzed areas, indicate high surface roughness of the struts. Root-mean-square deviation of the surface Sq reached 31,1 $\mu \mathrm{m}$. A negative skewness indicates the concentration of the material near the top surface, which can be associated with the agglomerates of particles adherent to the surface. Developed interfacial area ratio $\mathrm{Sdr}$ (defined as the ratio of the increment of the interfacial area of a surface over the sampling area) reaches an average value of $140 \%$. Analysis of the results presented in Table 3 indicates the existence of disparities between the calculated parameter values $\mathrm{Sdr}$ for different areas of the struts. For surfaces that are marked with the symbols a, b, c, h average (a)

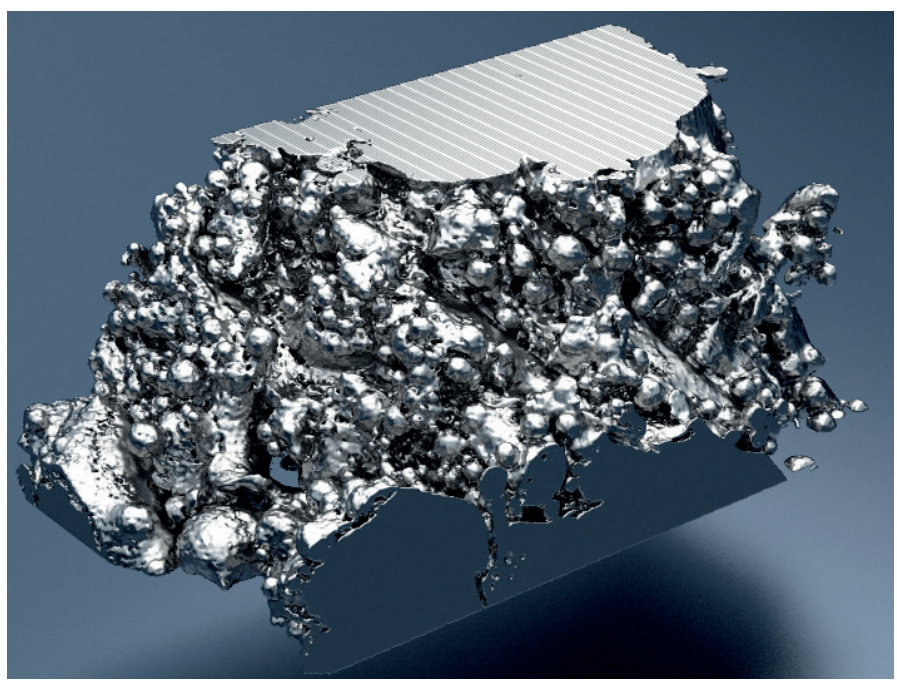

(b)

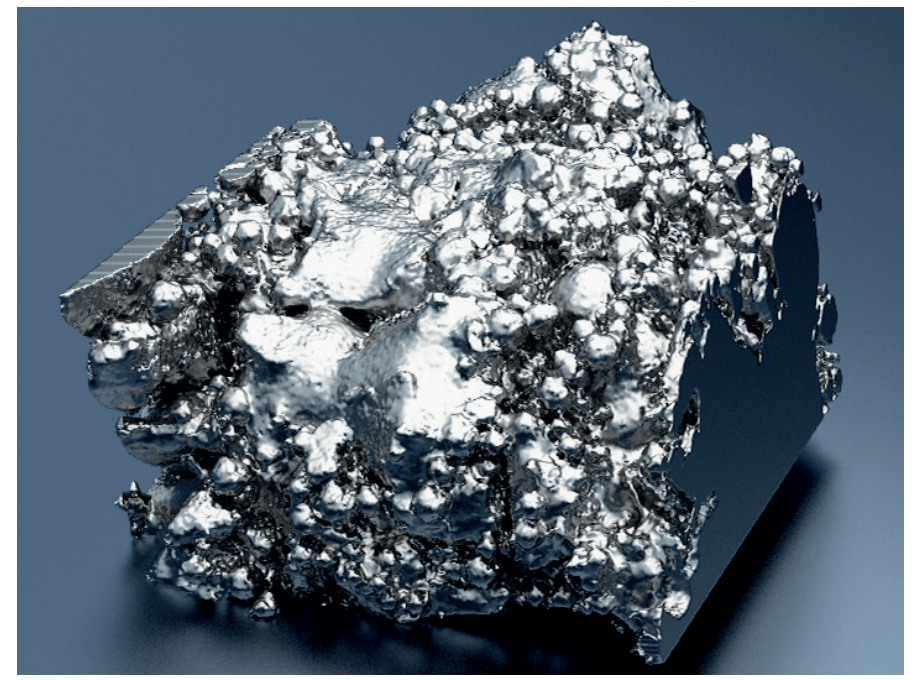

Fig. 5. Visualization of fragments of struts structure - X-ray computer microtomography 
(a)

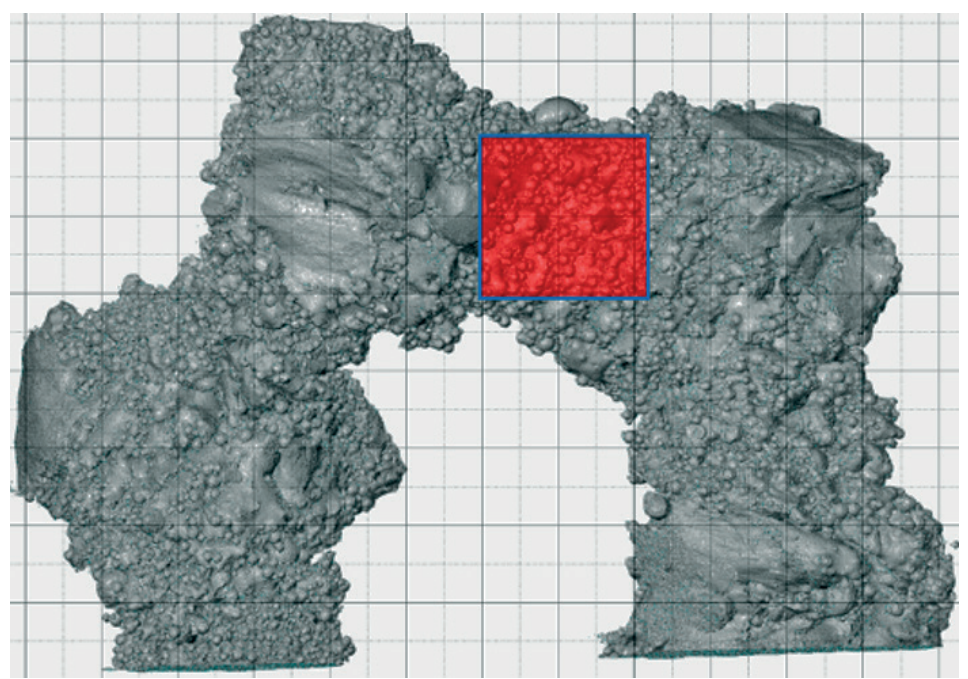

(b)

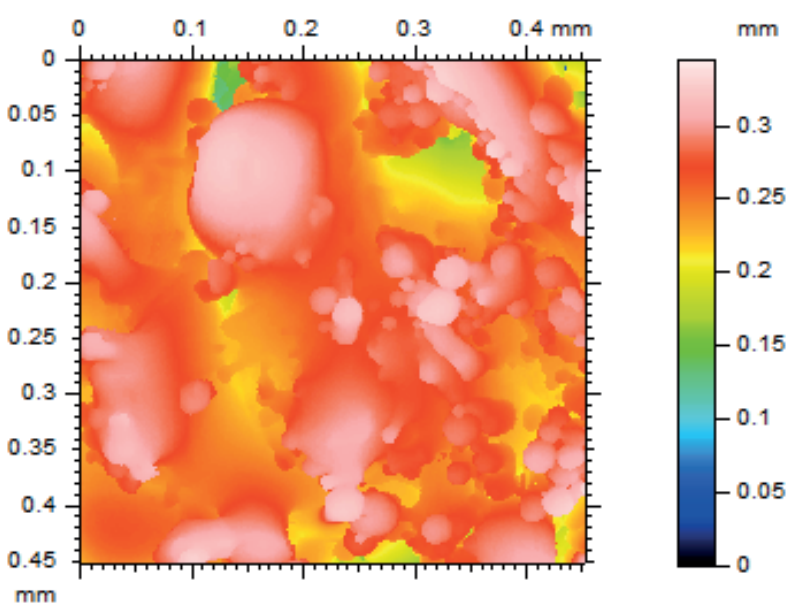

Fig. 6. A fragment of the struts in the XY view (a) and its contour map (b)
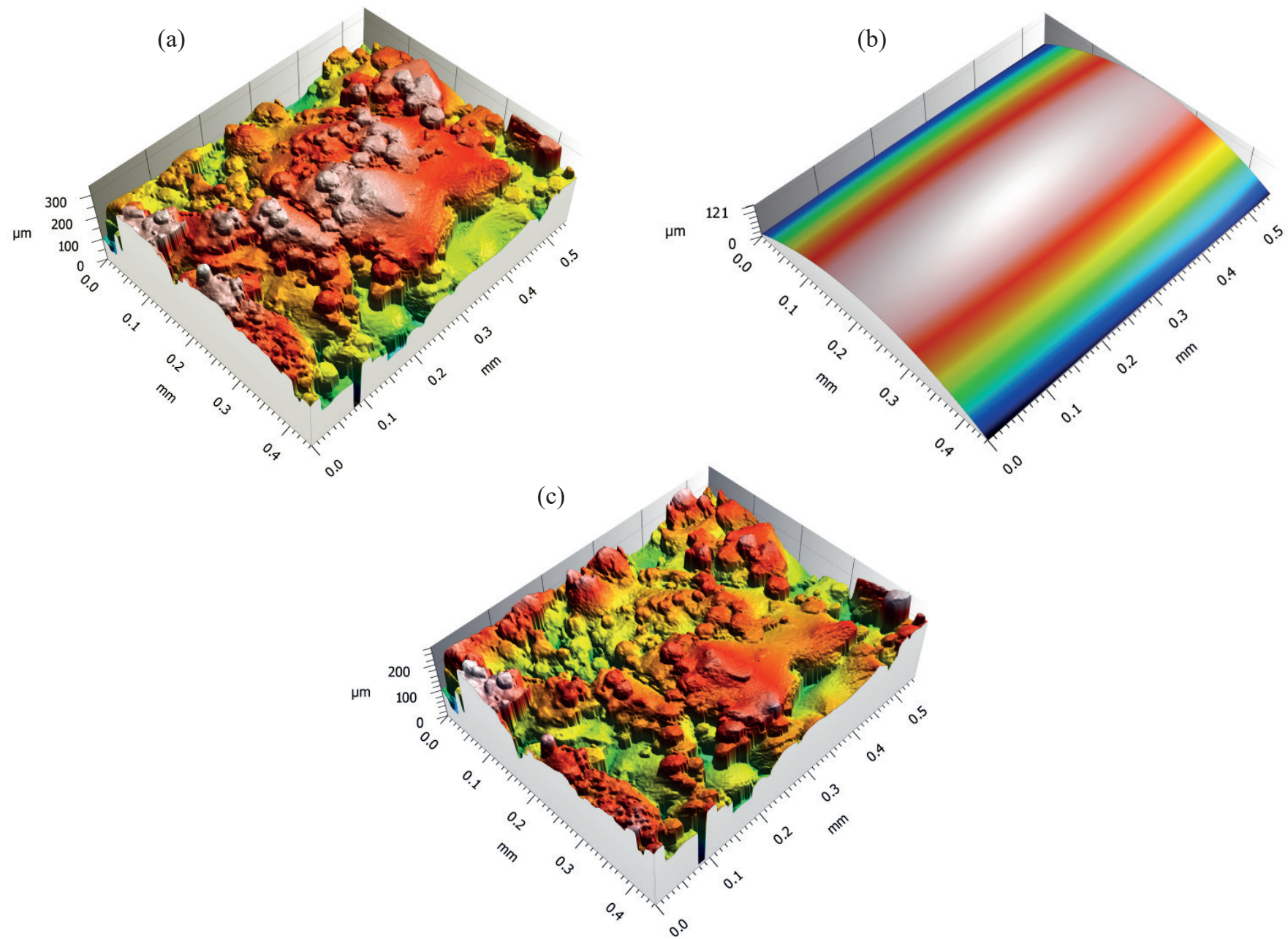

Fig. 7. Original measured surface (a), reference surface - second degree polynomial (b) and residual surface (c) 
J. Maszybrocka, A. Stwora, B. Gapiniskit, G. Skrabalak, and M. Karolus
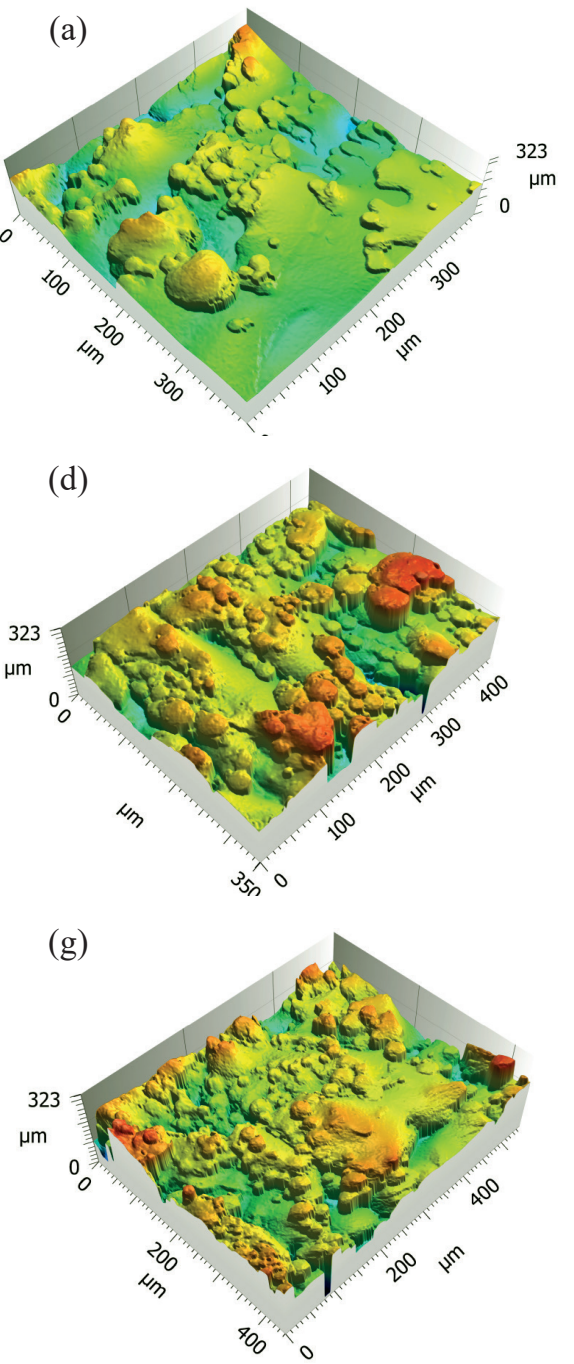
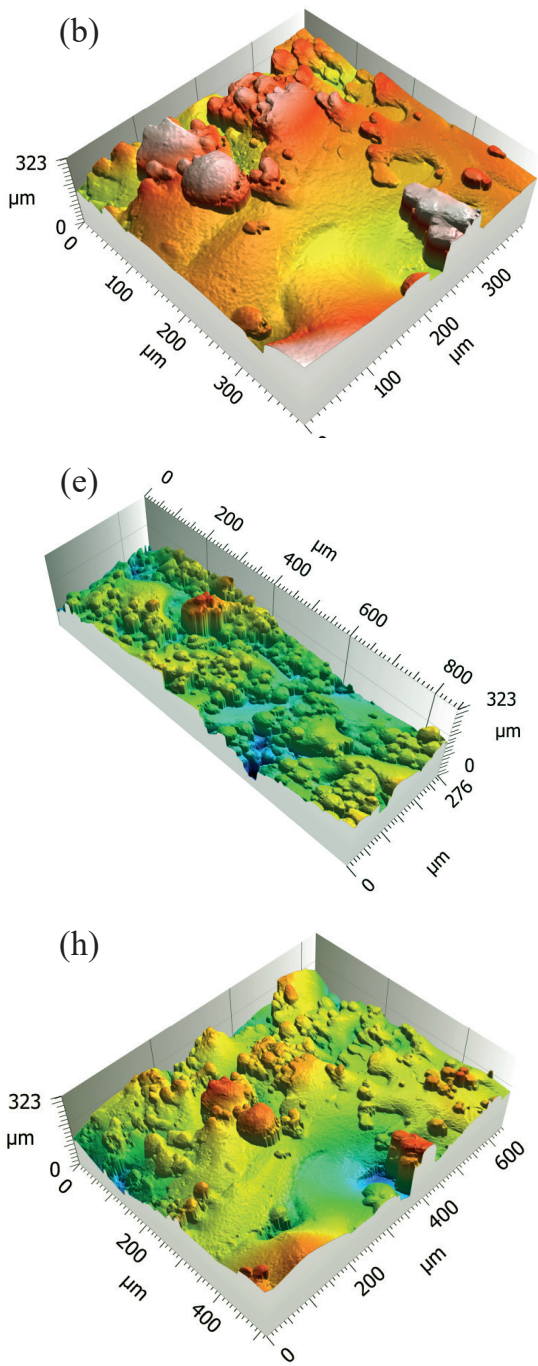
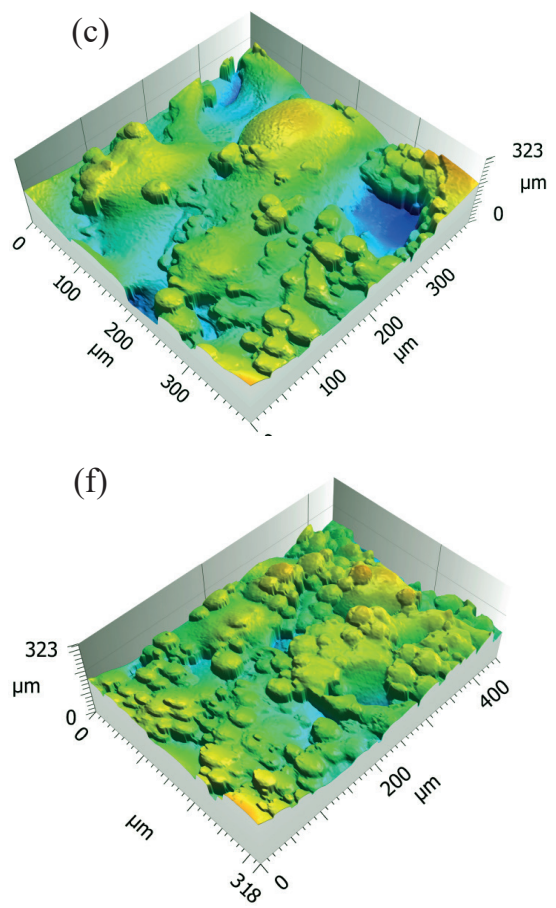

Fig. 8. Selected areas of the struts, which were analyzed

Table 3

Parameters determined for the surface from Fig. 8

\begin{tabular}{|c|c|c|c|c|c|c|c|c|}
\hline \multirow{2}{*}{ parametrs } & \multicolumn{7}{|c|}{ Amplitude parameters } & Hybrid parameter \\
\cline { 2 - 9 } & Sq & Ssk & Sku & Sp & Sv & Sz & Sa & Sdr \\
\hline \multirow{2}{*}{ (a) } & 26,9 & 0,002 & 3,15 & 85,8 & 149,0 & 234,8 & 21,6 & 128 \\
\hline (b) & 32,8 & 0,12 & 5,20 & 96,1 & 227,6 & 323,7 & 24,4 & 127 \\
\hline (c) & 34,0 & $-0,42$ & 3,34 & 89,8 & 135,1 & 224,9 & 26,6 & 121 \\
\hline (d) & 33,8 & $-0,70$ & 5,20 & 85,4 & 182,2 & 267,7 & 25,6 & 179 \\
\hline (e) & 32,3 & $-0,17$ & 4,20 & 113,4 & 142,3 & 255,7 & 25,2 & 144 \\
\hline (f) & 26,7 & $-0,91$ & 5,60 & 70,0 & 144,8 & 214,8 & 20,1 & 157 \\
\hline (g) & 30,2 & $-0,51$ & 3,80 & 88,6 & 175,6 & 264,2 & 24,0 & 162 \\
\hline (h) & 32,4 & $-0,34$ & 3,80 & 89,9 & 169,1 & 259,2 & 24,6 & 110 \\
\hline average value & 31,1 & $-0,37$ & 4,29 & 89,9 & 165,7 & 255,6 & 24,0 & 141 \\
\hline standard deviation & 2,9 & 0,35 & 0,93 & 12,1 & 30,3 & 33,6 & 2,1 & 23 \\
\hline
\end{tabular}


value of Sdr parameters was $121 \%$ and in case of the surface d, e, f, g 160\%. On 3d isometric views of surface topography with large values of the Sdr parameters can be seen spheres, which are in fact partially melted Ti6Al4V powders attached to the surface. The amount of particles attached to the surface depends on the location of the analyzed surface with respect to the production direction. This analysis quantitatively complements and confirms the conclusions from the qualitative analysis of SEM images of the struts surface.

\section{Summary}

One of the factors which influence the osseointegration processes of the implant is its surface roughness. Studies have shown that porous samples produced by selective laser sintering have a complex surface morphology of struts. Developed interfacial area ratio, evaluated on the basis of microtomography measurements, reaches a value of up to $180 \%$ and the mean square deviation of the surface $34 \mu \mathrm{m}$.

The applied method of analysis, based on tomography measurements allows the quantitative evaluation of surface topography of the struts, but the limitations of the methodology applied should be kept in mind. The transformation of point clouds to the rectangular grid in this case does not fully represent such a complex surface morphology of the struts.

Transformation can be applied to the edges with an angle of maximum $90^{\circ}$, and thus, the analysis of some points are not possible. This includes spherical particles which are attached to the bar and also to the calculation of the areas covered by the surface irregularities. Described problem associated with representation of surface topography does not also solve the application of other measurement techniques i.e. confocal and interferometry techniques. Although the measurement resolution would be improved, the illumination beam will also not be able to analyze a greater tilt than $90^{\circ}$. An important advantage of the method of using from CT scans is the ability to analyze the surface roughness of the struts in the entire volume of the porous structure without destroying it.

For quantitative analysis of surface topography, only the amplitude parameters and one hybrid parameter were selected. A more comprehensive characterization of surface topography can be obtained by analyzing other groups parameters and performing, e.g. motifs analysis. Fig. 9 shows an attempt at identification of spherical particles on the surface based on motifs analysis. This allows to determine the percentage of spherical particles attached to the surface. However, the limitations of the methodology applied require additional verification analysis, which will be the subject of further consideration.

The relatively high roughness is a consequence of the manufacturing process, during which the partially melted Ti6Al4V powders adhere to the surface of the struts. The degree of binding is so strong that they could not be removed during the cleaning process of the samples with compressed air and an ultrasonic cleaner. The limited ability to control the surface morphology at a micro scale during the manufacturing pro-

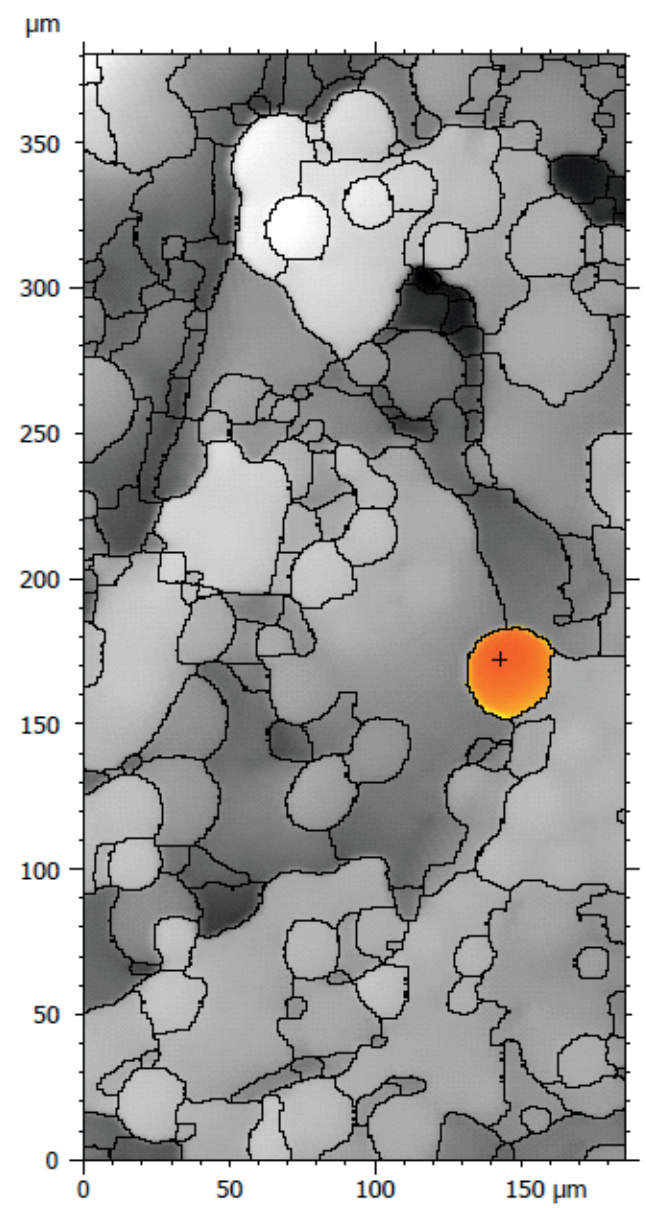

Fig. 9. Identification of spherical particles on the surface based on motifs analysis

cess by SLS makes it necessary to consider the introduction of additional implant surface modification treatment after its preparation.

Above all, appropriate surface treatment should remove partially connected, spherical particles from the surface. This is important when considering the use of Ti6Al4V lattice structure as part of the hip prosthesis stem. It is probable that during the implant use, mechanically detached spherical particles could migrate to the area of articulation. The particles move continuously, participating in the deleterious abrasive third-body wear process. As a consequence, they may be affecting the roughness of the femoral head and contributing to the increased wear of the polyethylene acetabular liner [20].

\section{REFERENCES}

[1] D.M. Brunette, P. Tengvall, M. Textor, P. Thomsen, Titanium in Medicine, Springer-Verlag, Berlin Heidelberg, 2001.

[2] D. R. Sumner, "Long-term implant fixation and stress-shielding in total hip replacement", Journal of Biomechanics 48(5), 797-800 (2015).

[3] P. A. Revell, Joint Replacement Technology, Woodhead Publishing in Materials, Cambridge, UK, 2008. 
[4] S. Sobieszczyk, The Development of Bioactive Porous Implants in Titanium Matrix, Publ. House Gdańsk Univ. Techn., Gdańsk, 2013.

[5] S. J Hollister, R. D. Maddox, J. M. Taboas, "Optimal design and fabrication of scaffolds to mimic tissue properties and satisfy biological constraints", Biomaterials 23(20), 4095-4103 (2002).

[6] T. Naoya, F. Shunsuke, T. Mitsuru, S. Kiyoyuki, O. Bungo, N. Takashi, M. Tomiharu, "Effect of pore size on bone ingrowth into porous titanium implants fabricated by additive manufacturing: An in vivo experiment", Materials Science and Engineering C: Materials for Biological Applications 59, 690-701 (2016).

[7] G. Ryan, A. Pandit, D. P. Apatsidis, "Fabrication methods of porous metals for use in orthopaedic applications", Biomaterials 27 (13), 2651-2670 (2006).

[8] N. Jha, D. P. Mondal, J. D. Majumdar, A. Badkul, A. Jha, A. K. Khare, "Highly porous open cell Ti-foam using $\mathrm{NaCl}$ as temporary space holder through powder metallurgy route", $\mathrm{Ma}$ terials \& Design 47, 810-819 (2013).

[9] D. C. Dunand, "Processing of titanium foams", Advanced Engineering Materials 6 (6), 369-376 (2004).

[10] Y. Chino, D. C. Dunand, "Directionally freeze-cast titanium foam with aligned, elongated pores", Acta Materialia 56(1), 105-113 (2008).

[11] J. P. Li, S. H. Li, C. A. Van Blitterswijk, K. De Groot, "A novel porous Ti6Al4V: characterization and cell attachment", Journal of Biomedical Materials Research Part A 73(2), 223-233 (2005).

[12] M. Wieczorowski, Surface Roughness Metrology, ZAPOL, Szczecin, 2013.
[3] M. Wieczorowski, Surface Topography Analysis, Publ. House Poznan Univ. Techn., Poznan, 2009.

[14] Krolczyk G., Raos P., Legutko S. Experimental analysis of surface roughness and surface texture of machined and fused deposition modelled parts, Tehnički Vjesnik - Technical Gazette 21 (1), $217-221$ (2014).

[5] G. Kerckhofs, G. Pyka, M. Moesen, S. Van Bael, J. Schrooten, M. Wevers, "High-resolution microfocus X-ray computed tomography for 3D surface roughness measurements of additive manufactured porous materials", Advanced Engineering Materials 15(3), 153-158 (2013).

[6] J. Krolczyk, B. Gapinski, G. Krolczyk, I. Samardzic, R. Maruda, K. Soucek, S. Legutko, P. Nieslony, Y. Javadi, L. Stas "Topographic inspection as a method of weld joint diagnostic", Tehnički vjesnik 23(1), 301-306 (2016).

[7] S. C. Kapfer, S. T. Hyde, K. Mecke, C. H. Arns, G. E. SchröderTurk, "Minimal surface scaffold designs for tissue engineering", Biomaterials 32(29), 6875-6882 (2011).

[8] C. Yan, L. Hao, A. Hussein, P. Young, "Ti-6Al-4V triply periodic minimal surface structures for bone implants fabricated via selective laser melting", Journal of the Mechanical Behavior of Biomedical Materials 5, 61-73 (2015).

[9] S. Van Bael, G. Kerckhofs, M. Moesen, G. Pyka, J. Schrooten, J. P. Kruth, "Micro-CT-based improvement of geometrical and mechanical controllability of selective laser melted Ti6Al4V porous structures", Materials Science and Engineering A528(24), 7423-7431 (2011).

[20] D. Najjar, M. Bigerelle, H. Migaud, A. Iost, "Identification of scratch mechanisms on a retrieved metallic femoral head", Wear 258 (1), 240-250 (2005) 